\title{
The synergystic effects of omega-3 fatty acids against 5 -fluorouracil-induced mucosal impairment in mice
}

\author{
Mayu Sebe ${ }^{1}$, Rie Tsutsumi ${ }^{1 *}$ (D), Sotaro Yamaguchi ${ }^{1}$, Yousuke T. Horikawa ${ }^{2}$, Nagakatsu Harada ${ }^{1}$, Takuro Oyama ${ }^{2}$, \\ Nami Kakuta ${ }^{2}$, Katsuya Tanaka ${ }^{2}$, Yasuo M. Tsutsumi ${ }^{2}$, Yutaka Nakaya ${ }^{1}$ and Hiroshi Sakaue ${ }^{1}$
}

\begin{abstract}
Background: Anti-cancer pharmaceuticals frequently have adverse side effects on patients such as gastrointestinal involvement limiting their clinical applications. These effects may be controlled by nutritional interventions, however, there are few studies that have shown any mechanistic effects. In this study, we examined effects of diet enhanced with eicosapentaenoic acid (EPA) and docosahexaenoic acid (DHA) on 5-fluorouracil (5-FU)-induced intestinal impairment and immunity in mice.

Methods: C57Bl6 mice were randomized to control diet, control diet + EPA, control + DHA, control + fish oil, or diet enchanced with DHA/EPA. After seven days of each respective diet, mice, excluding those in the sham group, were treated with $10 \mathrm{mg} / \mathrm{kg} /$ day 5 -FU for 7 days. The effects of 5-FU-induced impairment in the small intestine were assessed using cytokine concentrations in serum and tissue, secretory immunoglobulin (Ig) A, diamine oxidase (DAO) activity, the length of the small intestine, and the expression of apoptosis signaling genes.

Results: The EPA/DHA-enhanced diet resulted in the most beneficial, synergystic and protective effect against 5-FU induced weight loss. Protection against inflammation, impaired intestinal function, and immunity of the small intestine were also observed. Individually, a DHA-enriched diet demonstrated a protective effect against 5-FU damage with longer small intestine mucosal and crypt lengths, greater DAO activity, and higher IgA concentrations, whereas the EPA-enriched diet resulted in decreased inflammatory cytokine concentrations in both plasma and small intestine and expression of apoptosis target genes.
\end{abstract}

Conclusions: In conclusion, a diet enhanced with EPA and DHA results in synergism protecting against the detrimental effects of 5-FU and limiting chemotherapy induced mucosal impairment.

Keyword: Omega-3 fatty acids, 5-fluorouracil, Mucosal impairment, Small intestine

\section{Background}

The clinical application of anti-cancer drugs is limited by the frequent occurrence of severe side effects, including decreased immunity and patient discomfort [1-4]. Chemotherapy-induced intestinal impairment and gastrointestinal symptoms are often severe enough for care providers and patients to reconsider treatment options and many times stop optimal therapies. Chemotherapy induces not only morphological alternations in the gut but

\footnotetext{
* Correspondence: rtsutsumi@tokushima-u.ac.jp

${ }^{1}$ Department of Nutrition and Metabolism, Institute of Biomedical Sciences,

Tokushima University, 3-18-15 Kuramoto, Tokushima, Japan

Full list of author information is available at the end of the article
}

also impairs gut mucosal immunity [5-7]. Approximately $40 \%$ of patients undergoing chemotherapy, and up to $60 \%$ of those undergoing high-dose chemotherapy, develop mucositis, or severe inflammation and dysfunction of the gastrointenstial mucosa $[8,9]$.

Continuous infusion of 5-fluorouracil (5-FU), a fluorinated pyrimidine used in chemotherapy, has been shown to reduce gut-associated lymphoid tissue (GALT) mass and secretory IgA levels in mice model [7]. GALT is recognized as the center of systemic mucosal immunity. 5-FU treatment changes GALT mass and increase the risk of infectious complications in patients with 5-FU chemotherapy [10]. Inomata et al. demonstrated that a single oral dose of 
5-FU induces apoptosis and cell cycle arrest in intestinal crypts of BDF1 mice [11]. They also reported that 5-FU increases apoptosis and/or cell cycle arrest through systemic exposure, and p21 and BAX expression determines an individual cell's fate [12].

Nutrition is essential to maintain intestinal structure and function. A number of nutrients exert specific biological effects on both tumor and host biology [13]. Dietary fatty acids appear to have variable effect and protection against cancers. The long chain $n-3$ poly-unsaturated fatty acids (PUFAs) such as EPA (20:5 n-3) and DHA (22:6n-3) that occur in fish oil are considered protective. Whereas, $n-6$ PUFAs such as linoleic acid (18:2n-6), found in sunflower seeds and corn oil, have been associated with cancer acceleration/promotion in rodent models [14, 15], although more recent literature has refuted this hypothesis given no clinical correlations [16-18]. Reduced tumor incidence and multiplicity in rats has been observed in those fed $n-3$ PUFAs [19]. Protection against carcinogenesis by $n-3$ PUFAs may occur mediated by several mechanism including inhibition of inflammation [20].

In a rat model, $n-3$ PUFAs not only inhibited cancer development but also protected against 5-FU induced intestinal injury [6]. Gomez de Segura et al. reported that in the presence of 5-FU, supplementing the diet with DHA and protein prevented intestinal damage and the suppression of cellular apoptosis [6]. Interestingly, enteral formulas targeted for cancer patients include fish oil, which contains more EPA than DHA. This may be related to the reported anti-tumor effect of EPA. However, the differing effects of EPA and DHA on 5-FU induced intestinal impairment are not understood.

This study investigates the effect of a diet enriched with EPA- and DHA compared to the individual supplementation of fish oil, EPA, or DHA alone on 5-FU induced impairments in the gastrointestinal mucosa (e.g., lesions) and cellular immunity caused by GALT impairment. We hypothesized that EPA and DHA would provide additional synergistic protection against intestinal impairment.

\section{Methods}

Animals

All animals were treated in compliance with the Guidelines for Proper Conduct of Animal Experiment and Related Activities (Ministry of Education, Culture, Sports, Science and Technology of Japan) and the protocols, which was assigned to ARRIVE guidelines, approved by the Animal Care and Use Committee at the Tokushima University, Tokushima, Japan. Male C57BL/6NCrSlc mice (average body weight; $21.6 \pm 2.21 \mathrm{~g}, n=60$; SLC, Shizuoka, Hamamatsu, Japan) aged 8 weeks were purchased for the study. The mice were housed in an SPF air-conditioned room at $23 \pm 1{ }^{\circ} \mathrm{C}$ and $50 \pm 10 \%$ humidity with a 12-h light/dark cycle. The animals were given ad libitum access to their respective diets. Body weight and food intake of each mouse qt5was measured every other day. Each mouse was in single cage.

The mice were divided into 6 groups $(n=10$ each). The sham and control diets consisted of a 1:1 mix of Ensure $^{\bullet}$ and Ensure $\mathrm{Hi}^{\circ}$; Abbott Japan, Tokyo, Japan (Sham and Cont groups, respectively). Three other groups with the control diet were then supplemented via oral gavage of $20 \mathrm{~g} / \mathrm{kg} /$ day body weight fish oil (FO; Sigma-Aldrich Japan, Tokyo, Japan), 2,000 mg/kg/day EPA (Wako Pure Chemical Industries, Osaka, Japan), or $2,000 \mathrm{mg} / \mathrm{kg} / \mathrm{day}$ DHA (Sigma Aldrich Japan, Tokyo, Japan). A final diet fortified with EPA and DHA was used (Prosure; ${ }^{\circ}$ Abbot Japan, Tokyo, Japan). The composition of the control and enhanced diets are provided in Table 1 . After 7 days of the allocated diet, daily injections of intraperitoneal 5-FU (10 $\mathrm{mg} / \mathrm{kg}$ body weight) were administered for 7 more days; the sham group received daily injections of $0.1 \mathrm{~mL}$ saline instead. Primary experimental outcomes are survival and weight changes.

\section{Plasma and tissue isolation}

Following completion of the 5-FU injections mice were euthanized by pentobarbital and blood was immediately collected from the right ventricle of the heart using a heparinized syringe and placed on ice. Plasma was separated by centrifugation at $5,000 \mathrm{~g}$ for $5 \mathrm{~min}$ at $4{ }^{\circ} \mathrm{C}$. Blood samples were frozen at $-80^{\circ} \mathrm{C}$. We took the small

Table 1 Main dietary compositions per $80 \mathrm{~mL}$

\begin{tabular}{lll}
\hline Nutrients & CONT & EPA/DHA-rich \\
\hline Calories, kcal & 100 & 100 \\
Protein, g & 3.52 & 5.33 \\
Protein sources & Casein Na, Soy protein & Casein Na, Whey protein \\
CHO, g & 13.728 & 16.3 \\
CHO sources & Dextrin, Sucrose & Dextrin, Sucrose \\
Dietary fiber, g & - & 0.77 \\
Fat, g & 3.52 & 4 \\
EPA, mg & - & 352 \\
DHA, mg & - & 160 \\
Fat sources & Corn oil & Fish oil, MCT, rapeseed oil \\
n-6:n-3 ratio & $4.36: 1$ & $1: 3.56$ \\
water, mL, \% & 68.45 & 63.5 \\
Vitamin E, mg & 3.0 & 16.0 \\
Vitamin C, mg & 15.2 & 34 \\
Zinc, mg & 1.5 & 2.0 \\
Iron, mg & 0.9 & - \\
Copper, mg & 0.1 & - \\
Manganese, mg & 0.2 & - \\
Magnesium, mg & 20 & 33 \\
\hline
\end{tabular}


intestine and removed the duodenum, and took $10 \mathrm{~cm}$ of upper (duodenum) side of intestine as jejunum. We used this jejunum as a small intestine and washed for IgA assay first, and upper $2 \mathrm{~cm}$ of jejunum was frozen and stored at $-80{ }^{\circ} \mathrm{C}$ for gene expression analysis. second $2 \mathrm{~cm}$ of it was stored in $4 \%$ paraformaldehyde for tissue stain to assess the length of small intestine.

\section{ELISA assays of IL- 6 and TNF- $a$}

Serum IL- 6 and TNF- $\alpha$ concentrations were measured using Mouse IL-6 and TNF- $\alpha$ Quantikine ELISA kits (R\&D Systems, Minneapolis, MN, USA). Briefly, the assay diluent was added to each well of a 96-well plate, followed by serum samples and the standard; the solutions were incubated for $2 \mathrm{~h}$ at room temperature. After each well was washed 5 times, the solutions were incubated with $100 \mu \mathrm{L}$ of mouse IL- 6 or TNF- $\alpha$ conjugate for $2 \mathrm{~h}$ at room temperature. The wells were washed 5 times again and then incubated with the substrate solution for $30 \mathrm{~min}$ at room temperature while protected from the light. Stop solution $(100 \mu \mathrm{L})$ was added to each well, and the optical density was determined at $450 \mathrm{~nm}$ using a microplate reader (Tecan Japan, Kanagawa, Japan).

\section{Gene expression analyses}

Total RNA was extracted from the small intestine using RNeasy Plus Universal Mini Kits (QIAGEN, Valencia, CA, USA). Total RNA ( $1 \mu \mathrm{g})$ was reverse-transcribed to cDNA in a final volume of $20 \mu \mathrm{L}$ using the Primescript RT Reagent kit (Takara, Shiga, Japan). Real-time PCR was performed in a final volume of $10 \mu \mathrm{L}$ containing $50 \mathrm{ng}$ of the cDNA template and primers using SYBR green and a StepOnePlus Real-Time PCR System per manufacturer's preset protocols (Life Technologies, Carlsbad, CA, USA). All samples were run in triplicate and a melting curve was done to verify sample purity. Data was analyzed per fold change using comparative $\mathrm{Ct}(\Delta \Delta \mathrm{Ct})$.

To determine the effect on inflammatory cytokines gene expression, we measured the expression of the IL- 6 and TNF $\alpha$ genes. To determine the effect on apoptosis gene expression, we measured the expression of the BH3interacting domain death agonist (BID), Bcl-2-associated death promoter (BAD), Caspase-3, Caspase-8, and Caspase-9, Bcl-2 associated X protein (BAX) and Fas cell surface death receptor (FAS) genes. To determine the effect on anti-apoptosis gene expression, we measured the expression of the B-cell lymphoma 2 (BCL-2) and inhibitor of apoptosis 1 (IAP-1) genes.

\section{Apoptosis}

Apoptosis was measured via terminal deoxynucleotidyl transferase-mediated dUTP nick-end labeling (TUN + EL) assays. Small intestines previously washed with PBS and fixed in $3.7 \%$ formalin, were sectioned $(5 \mu \mathrm{m})$. TACS ${ }^{\odot} 2$
TdT DAB (diaminobenzidine) Kit (Trevigen, Gaithersberg, MD, USA) was used according to the manufacturer's instructions. Briefly, intestinal tissue was washed and incubated in proteinase $\mathrm{K}$ for $15 \mathrm{~min}$ at room temperature. The reaction was quenched and then washwed with PBS. Apoptotic cells were lableled with TdT for $5 \mathrm{~min}$. Samples were again washed and visualized using strep-HRP solution for approximately $5 \mathrm{~min}$. Samples were then counterstained with $1 \%$ methyl green for approximately $2 \mathrm{~min}$.

\section{Diamine oxidase activity}

Diamine oxidase (DAO) activity was measured by ELISA using mice serum from Shizen-Meneki Oyo Giken Co. (Takamatsu, Kagawa, Japan) using a previously reported protocol [21]. Briefly, plasma was added to a cadaverine solution and incubated. The incubation mixture was then mixed with a color reagent containing DA-67 and peroxidase. After a given period, the absorption of the reaction product was measured colorimetrically at $668 \mathrm{~nm}$ against the blank solution using a spectrophotometer. The plasma DAO activity is expressed as units/L.

\section{$\lg \mathrm{A}$ quantification}

IgA was measured from the intestinal washings using a Mouse IgA ELISA Quantitation Set (Bethyl Laboratories, Inc., Montgomery, TX, USA). Briefly, the plate was coated with goat anti-mouse IgA for $1 \mathrm{~h}$, blocked with blocking solution for $30 \mathrm{~min}$, and incubated with the standard (mouse reference serum) or washing solution at room temperature for $1 \mathrm{~h}$. Incubation with HPRconjugated antibody was performed for another $1 \mathrm{~h}$. The incubation solution was then mixed with $3,3^{\prime}, 5,5^{\prime}$-tetramethylbenzidine (TMB) substrate solution, and the absorbance was measured at $450 \mathrm{~nm}$ using a microplate reader (Tecan Japan, Kanagawa, Japan).

\section{Statistical analysis}

All data are expressed as mean \pm SD. Statistical significance $(P<0.05)$ was determined using ANOVA followed by the Tukey's or Bonferroni post hoc test with GraphPad Prism5 (San Diego, CA, USA).

\section{Results}

EPA-DHA rich diets minimize 5-FU related weight loss

There were no significant differences between the groups in body weight while acclimating to their respective diets (Fig. 1a, b). All treatment groups after 5-FU lost weight. However, EPA-DHA-rich $(-0.47 \pm 0.59 \mathrm{~g}, N=7, P<0.001)$ diets or Cont + FO $(-0.83 \pm 1.05 \mathrm{~g}, N=7, P<0.001)$ diets had significantly less weight loss when compared to Cont $(-2.7 \pm 0.42 \mathrm{~g}, N=7)$ diet alone. Furthermore, no protection against 5-FU induced weight loss was observed in Cont + EPA $(-2.85 \pm 0.41 \mathrm{~g}, N=7, N . S$. vs. Cont $)$ or Cont + DHA (-3.61 \pm 0.89 g, $N=7, N . S$. vs. Cont) diets alone 


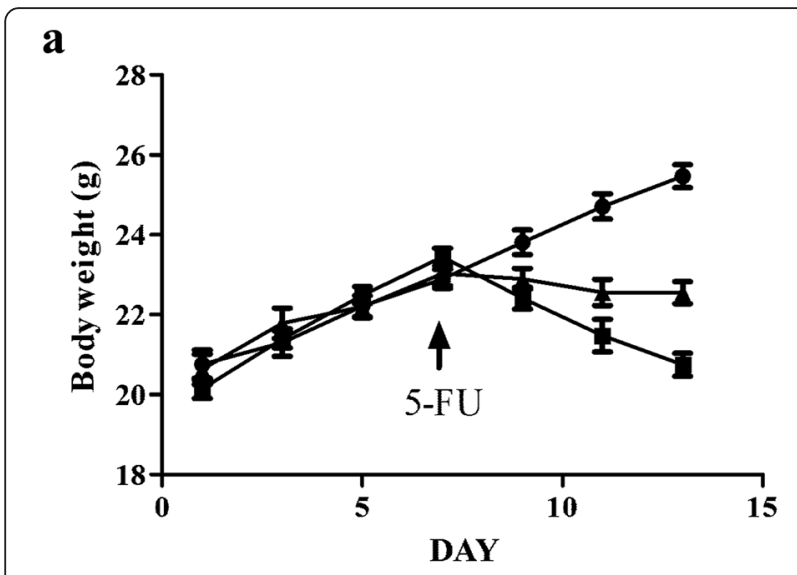

b
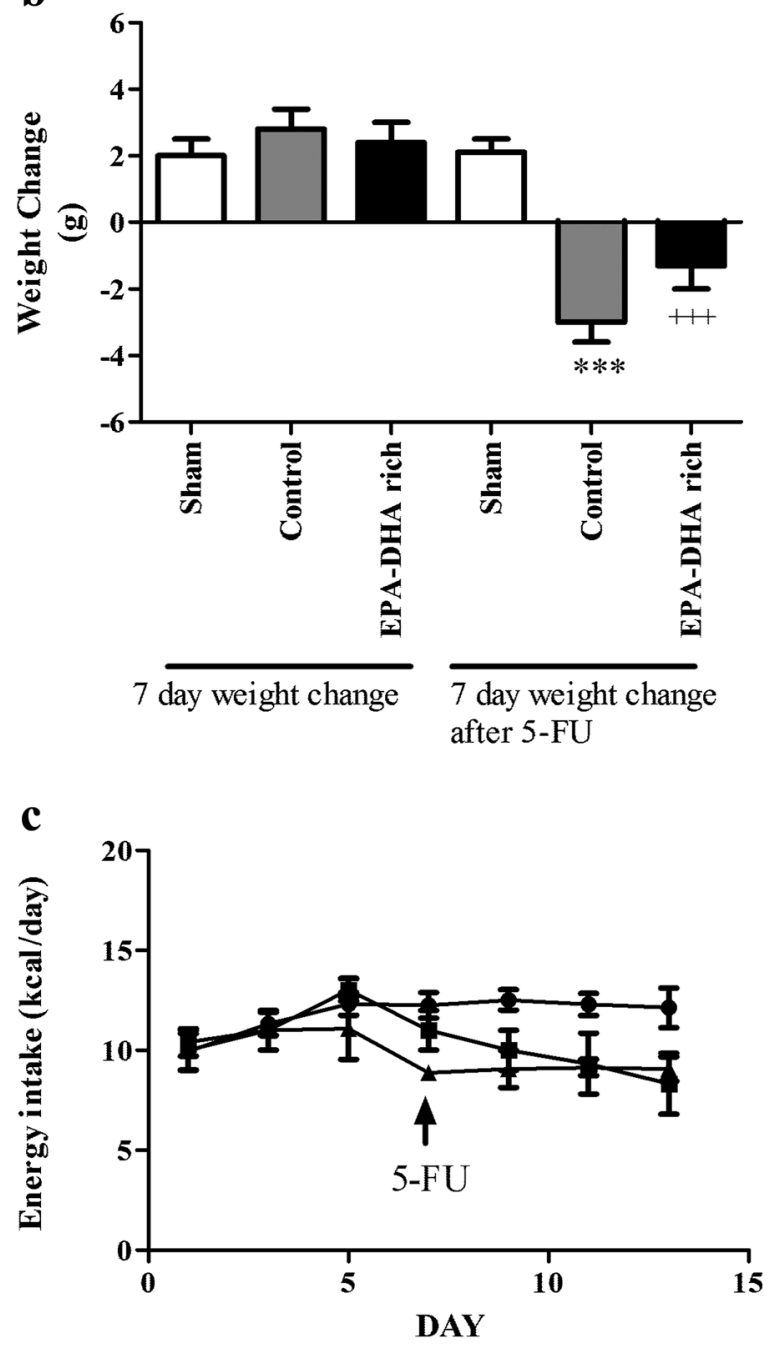

Fig. 1 An eicosapentaenoic acid (EPA) and docosahexaenoic acid (DHA) rich diet minimizes weight loss associated with 5-Fluorouracil (5-FU). a Body weight changes observed in mice during diet introduction and while on 5-FU. $\mathbf{b}$ Weight gain and loss after 7 days of 5-FU chemotherapy. c Food intake changes in mice throughout the study. C57BL/6 mice were fed their respective diets, as shown, with 7 days of daily intra-peritoneal injections of $10 \mathrm{mg} / \mathrm{kg}$ body weight 5-FU. Values are presented as mean \pm SD. $N=7$ per experimental groups. ${ }^{* *} P<0.001$ vs control (cont); $+++P<0.001$ vs control. FO, fish oil

(Fig. 1a, b). Although mice displayed significant weight loss, no significant change in food ingestion were observed, as shown in Fig. 1c, during 5-FU injections.

\section{EPA-DHA rich diets prevent inflammatory cytokine activation and expression}

As illustrated in Fig. 2a and b, the 5-FU injections resulted in significantly higher serum concentrations of inflammatory cytokines IL- 6 and TNF- $\alpha$, compared to the sham group $(P<0.01)$. The EPA-DHA-rich diet had significantly lower serum concentrations of IL-6 (105 $\pm 45 \mathrm{pg} / \mathrm{mL}, N=7, P<0.5)$ and TNF- $\alpha(168 \pm 61 \mathrm{pg} / \mathrm{mL}$, $N=7, P<0.5)$ compared to Control (IL-6:181 $\pm 68 \mathrm{pg} / \mathrm{mL}$ and TNF- $\alpha$ : $323 \pm 141 \mathrm{pg} / \mathrm{mL}, N=7)$. Interstingly, the Cont + EPA diet resulted in significantly lower concentrations of IL-6 $(107 \pm 37 \mathrm{pg} / \mathrm{mL}, N=7, P<0.5)$ but no significance was observed in TNF- $\alpha(190 \pm 114 \mathrm{pg} / \mathrm{mL}$, $N=7$, N.S.).

Relative gene expression of IL-6, within the small intestine was similar to sham treated animals in both EPADHA rich and Cont + EPA groups, with a 1.5-2 fold increase observed in the other remaining diets. An increase in TNF- $\alpha$ gene expression was observed in all groups following 5-FU exposure although, EPA-DHA rich and Cont + EPA diets had relatively lower expression levels than the other diets (Fig. 2c and d).

EPA-DHA rich diets minimially affect apoptotic genes, but upregulate anti-apoptotic genes

As shown in Fig. 3a, all mice given 5 -FU had a releative increase in pro-apoptotic genes as expected given that chemotherapeutic agents target cellular death. EPA$\mathrm{DHA}$ rich, Cont $+\mathrm{FO}$, and Cont + EPA diets were all equally able to reduce relative expression of the apoptotic genes: BH3-interacting domain death agonist (BID), Caspase-3, Caspase-8, and Caspase-9, although minimal effects were noticed in the other apoptotic genes.

Control and Cont + DHA fed mice had no relative change within anti-apoptotic gene expression, Whereas, EPA-DHA-rich, Cont + FO, and Cont + EPA diets resulted in greater expression of both BCL-2 and IAP-1 genes., which is likely one of the mechanisms involved in omega-3 induced intestinal protection. 

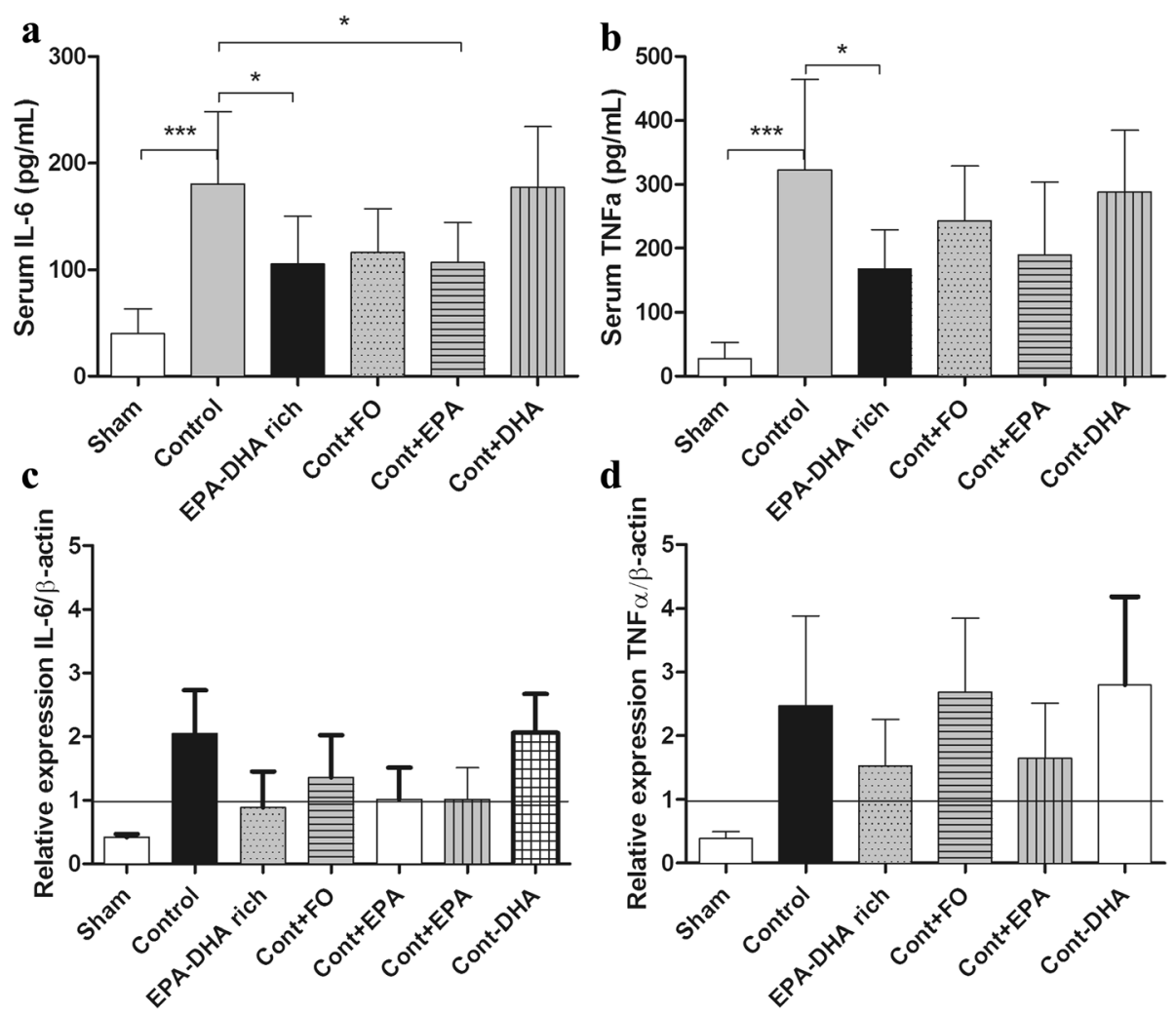

Fig. 2 An eicosapentaenoic acid (EPA) and docosahexaenoic acid (DHA) rich diet limits inflammatory cytokine release and gene expression within the small intestine following 5-Fluorouracil (5-FU). Serum concentrations of IL-6 (a) and TNF-a (b) were measured. Relative gene expression ( $\triangle \Delta C \mathrm{Ct})$ within the small intestine were also measured for IL-6 (c) and TNF-a $(\mathbf{d})$ C57BL/6 mice were fed their respective diets and them treated with 7 days of $10 \mathrm{mg} / \mathrm{kg}$ body weight intra-peritoneal 5-Fluorouracil or $0.1 \mathrm{~mL} /$ day saline (sham). Values are expressed as mean \pm SD. $N=7$ per experimental groups. ${ }^{*} P<0.05$ and ${ }^{* * *} P<0.001$. FO, Fish oil. IL-6, interleukin-6. TNF-a, tumor necrosis factor alpha

TUNEL staining of the intestines resulted in similar results (Fig. 3b). The EPA-DHA rich diet had significantly lower TUNEL positive cells compared to Control $(7.3 \pm 2 \%$ vs. $14.9 \pm 3 \%, N=7, P<0.001)$, Cont + FO (12.6 $\pm 3 \%, N=7, P<0.01)$, and Cont + DHA $(18.4 \pm 4 \%, N=7, P<0.001)$. EPA-DHA rich diet resulted in no change in TUNEL positive cells when compared to Sham or Cont + EPA.

\section{EPA-DHA rich diet maintained intestinal integrity and permeability}

Following 5-FU injections, gross histological sections of the small intestine revealed inflammation and disrupted mucosal architecture in Control, Cont $+\mathrm{FO}$, Cont + EPA, and Cont + DHA (Fig. 4a). However, EPA-DHA rich diet fed mice exhibited intestinal mucosa similar to Sham animals suggesting preserved intestinal mucosa and integrity. This was further quantified by intestinal mucosal villi and crypt length (Fig. 4b, c). EPA-DHA rich diets revealed significantly longer villi when compared to Control $(458 \pm 62 \mu \mathrm{m}$ vs. $310 \pm 89 \mu \mathrm{m}, N=7, P<0.05$, respectively), Cont + FO $(317 \pm 85 \mu \mathrm{m}, N=7, P<0.05)$ or Cont + EPA $(292 \pm 91 \mu \mathrm{m}, N=7, P<0.05)$ diets, likely resulting in preserved intestianl function and permeability. Interestingly, there was no significant difference among crypt length.

5-FU treatment significantly decreased DAO activity in all groups except for the EPA-DHA rich diet group. Furthermore, EPA-DHA rich group maintained significantly higher DAO activity then Control (Fig. 4d; $4.3 \pm 1.1 \mathrm{U} / \mathrm{L}$ vs. $2 \pm 0.8 \mathrm{U} / \mathrm{L}, N=7, P<0.05$, respectively).

\section{EPA-DHA rich diets preserves intestinal immunity}

Intestinal IgA concentrations were significantly lower in the groups injected with 5-FU (Fig. 5). However, mice fed the EPA/DHA-rich diet had significantly higher IgA concentrations compared to Control diet $(119 \pm 25 \mu \mathrm{g} / \mathrm{mL}$ vs. $79 \pm 21 \mu \mathrm{g} / \mathrm{mL}, N=7, P<0.05$, respectively suggesting preserved immune function. There were no significant differences in IgA concentrations between the Control, Cont + FO, Cont + EPA, or Cont + DHA.

\section{Discussion}

Mucositis, especially along the gastrointestinal tract remains a significant problem for cancer patients 


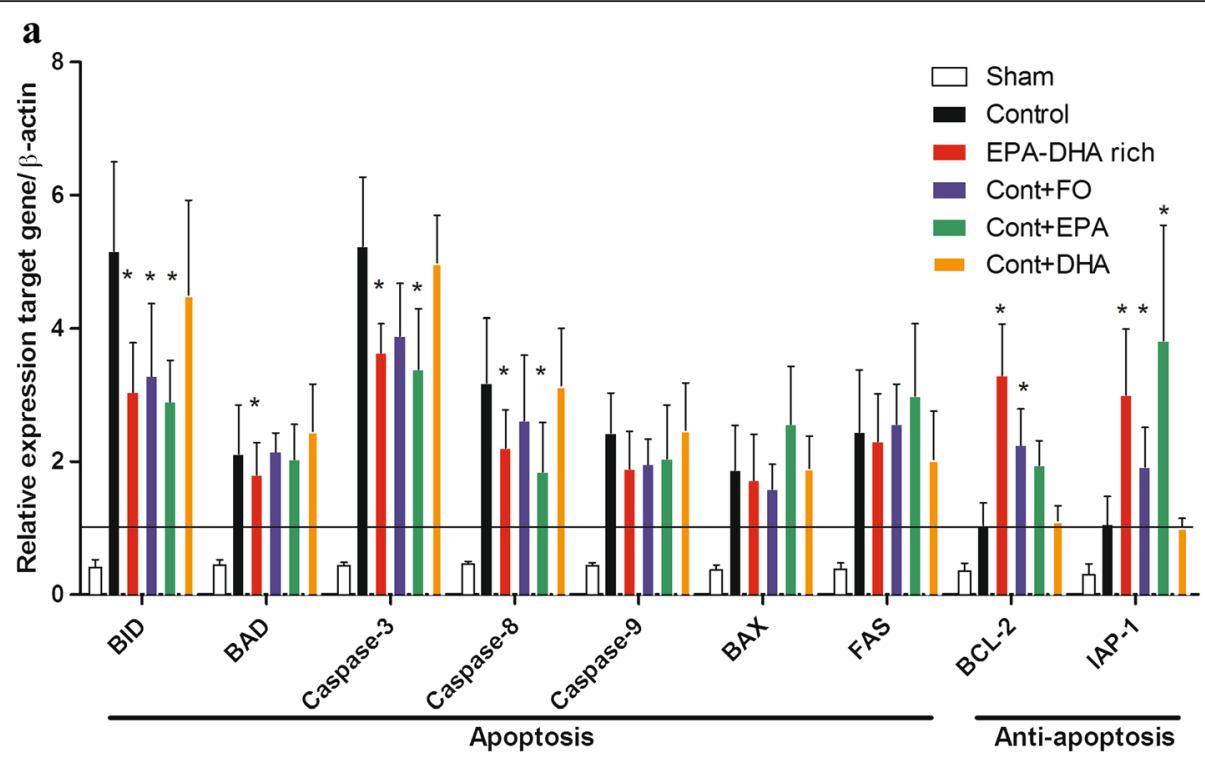

b

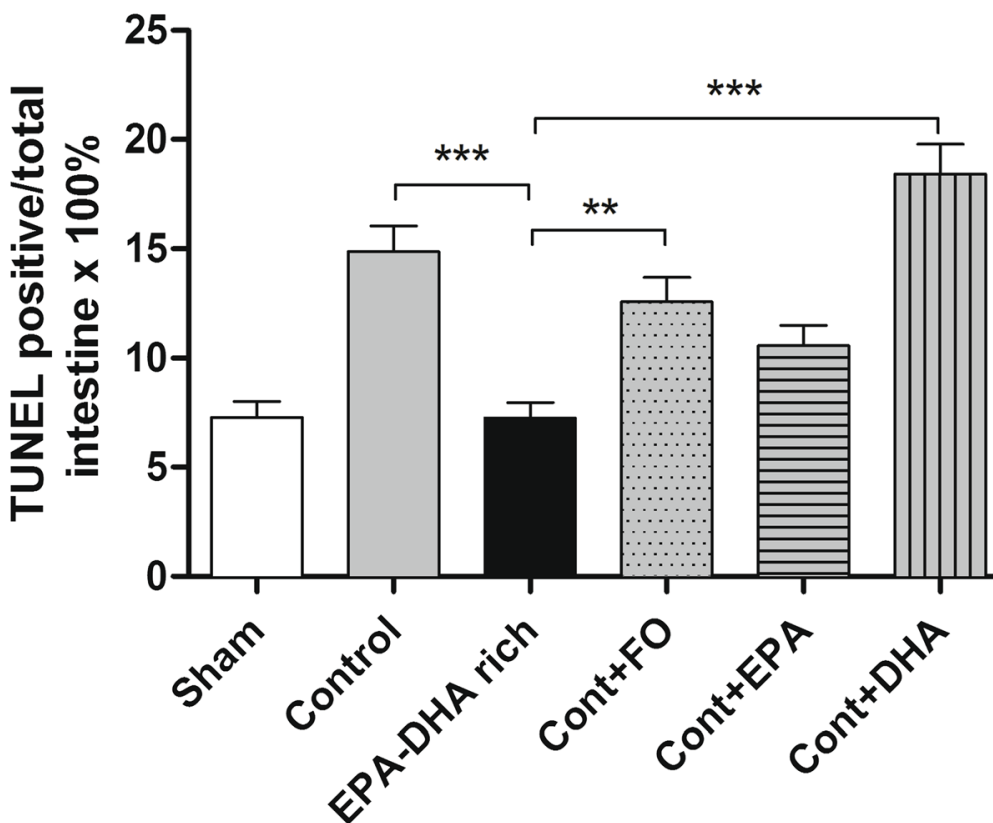

Fig. 3 Eicosapentaenoic acid (EPA) and docosahexaenoic acid (DHA) affect apoptosis. a Gene expression of apoptosis and anti-apoptosis genes following 7 days of 5 -fluorouracil. $\mathbf{b}$ Quantitative analysis of TUNEL positive cells. Values are expressed as mean \pm SD. $N=7$ per experimental groups. ${ }^{* *} P<0.01$ and ${ }^{* * *} P<0.001$. BAD, BCL-2-associated death promoter; BCL-2, B-cell lymphoma 2; BAX, BCL-2-associated X protein; BID, BH3-interacting domain death agonist; IAP-1, inhibitor of apoptosis 1

undergoing chemotherapy. In the present study, we demonstrated a beneficial effect of omega-3 PUFAs as well as a synergystic effect of an EPA/DHA-rich diet on 5-FU induced impairment of small intestine function and immunity. However, together, EPA and DHA were able to significantly limit the cytotoxic damage observed by 5-FU.

Daily intake of an EPA/DHA-rich diet resulted in less weight loss in the presence of 5-FU than the other diets without a difference in food intake and caloric levels. This is likely due to preserved intestinal function. As EPA-
DHA rich group is protected against chemotherapy induced intestinal injury, they are able to easily absorb nutrients and continue to metabolize and grow. Anticancer drug-induced derangements of the intestinal barrier may pass through the gut wall and it might induce systemic inflammation and infection [10] as well as poor nutritional uptake resulting in malnutrition and weight loss. Nagayoshi et al. demonstrated that even small doses of 5FU caused neither diarrhea nor anorexia, and markable cell loss in GALT effector sites [7]. GALT works as a gut mucosal immunological barrier and as a critical tissue in 


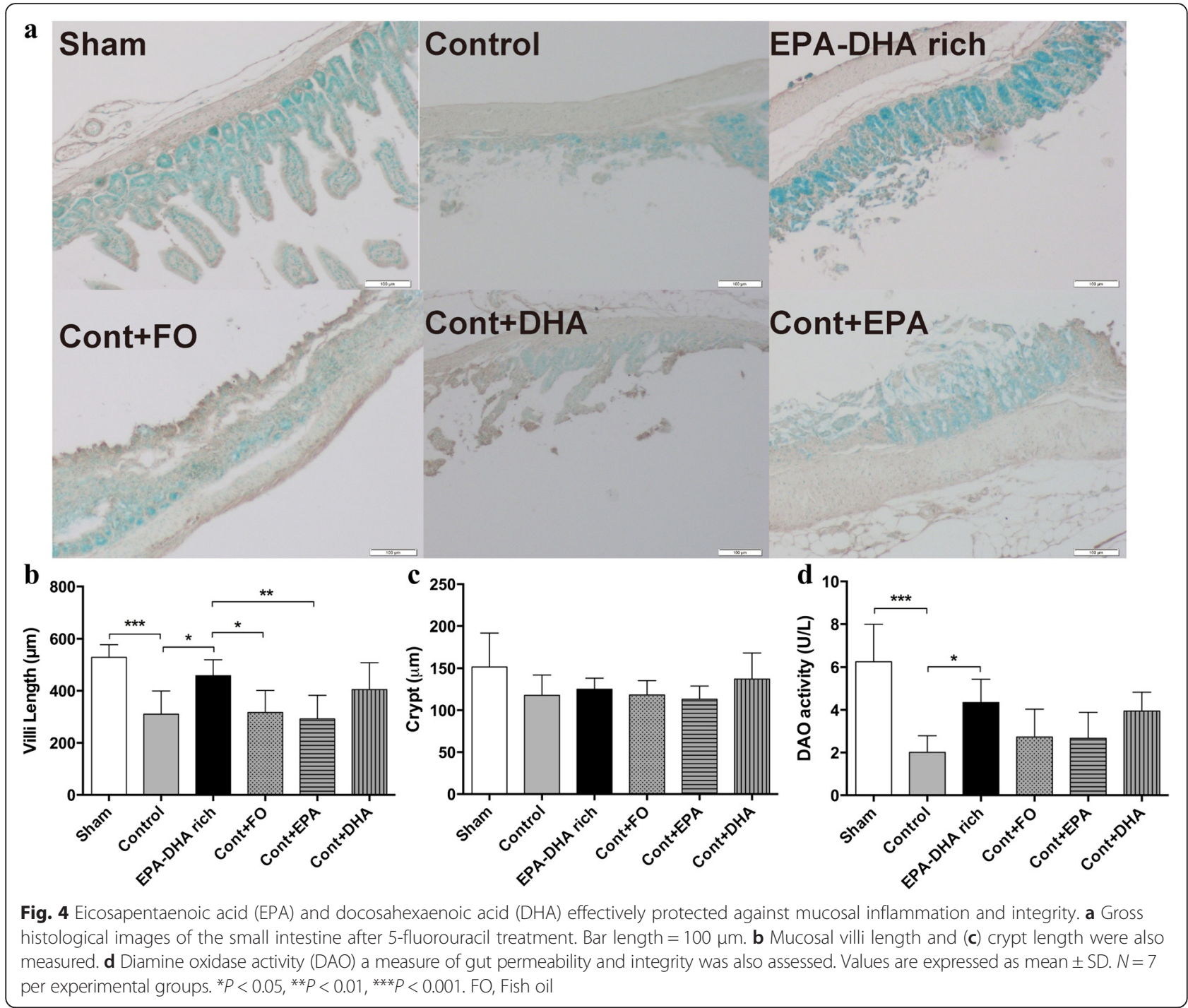

mucosal immunity [22]. In the present study, secretory IgA concentrations were lower in the 5-FU treated groups, and the EPA/DHA-rich diet resulted in higher IgA concentrations than the other diets, suggesting a protective and preserved effect on intestinal immunity. In this study, we measured IgA in intestinal washing according to our previous work [23] but could not provide the variability of the assay in this matrix.

It will be suggested that increase of crypt cell proliferation protected mucosa (vill) and crypt length [24]. Tazuke et al. demonstrated an increase in small intestinal crypt cell proliferation following glutamine administration in a rat model of chemotherapy (cisplatin)-induced mucosal injury [25]. In the present study, dietary enrichment with EPA and DHA provided protection against intestinal mucosa and crypt damage caused by 5 -FU, while fish oil and EPA supplementation did not have the same effects. Consistent with these results, DAO activity, which is an indicator of gut inflammation and integrity, was lower in the groups with 5-FU treatment, and, among the diet groups, was highest in the group receiving the EPA/DHArich diet. Therefore, the data suggest that DHA may play a role in protecting the small intestine from chemotherapeutic agents.

The combined EPA-DHA diet contained less EPA and DHA than the diets supplemented with FO, DHA, or EPA alone, however, the combined EPA/DHA diet resulted in the greatest protection of the small intestine. Although this is likely related to the synergistic effects of EPA and DHA together there may be that another component of the diet that may have contributed to the effect. Previous studies have demonstrated the effect of a hyper-proteic diet in the protection against intestinal mucosa damage which was confirmed after 5-FU treatment [26, 27]. Furthermore, we previously reported that whey-based protein diets have also been shown to be protective [28]. The 


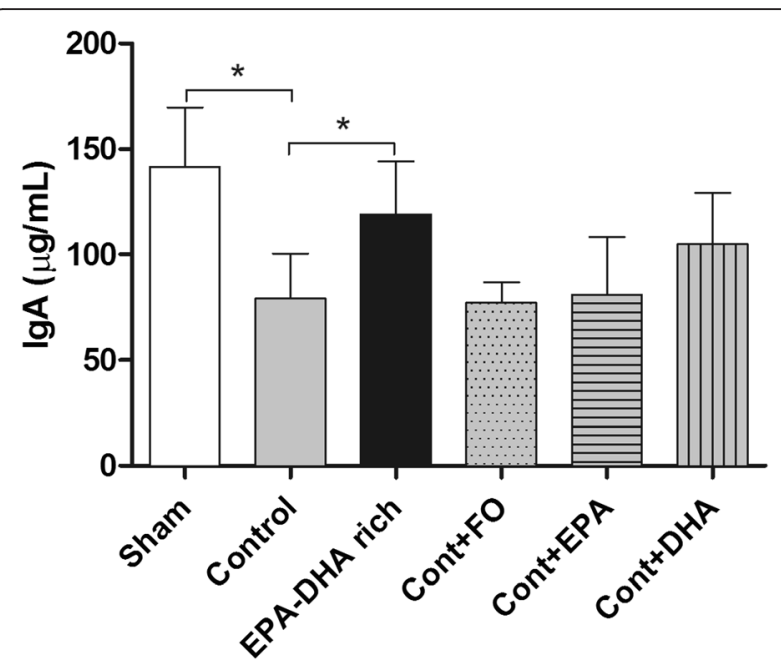

Fig. 5 Eicosapentaenoic acid (EPA) and docosahexaenoic acid (DHA) maintained intestinal immunity after 5-Fluorourcil treatment. Secretory IgA concentrations were measured by from the small intestines. Values are expressed as mean \pm SD. $N=7$ per experimental groups. ${ }^{*} P<0.05$. FO, Fish oil

addition of amounts of specific nutrients, such as glutamine and arginine, would also induce benefits for intestinal structure and function $[29,30]$. These are considered that are important for enterocyte development and may have protective effects against radiation damage [31].

According to a report from Gomez de Segura et al., the dietary proteins and DHA act complementary, and their combined use would be acceptable. This joint administration of both nutrients, i.e. protein and DHA, improve morphologic change and apoptosis in the intestine compared to the effects of either nutrient alone [6]. In fact, we observed different effects of each diet on apoptosis, and the administration of DHA alone caused a rise in apoptosis, confirming the pro-apoptotic effect attributed to this fatty acid [32].

The benefit of DHA supplementation could not be detected with standard diet in rat with intestinal impairment by 5-FU and administration of supplementary protein is also needed to protect intestinal lesions [6]. Geske et al. suggested that DNA recovery is induced early in p53-induced apoptosis [33]. This early apoptosis by $\mathrm{p} 53$ also leads to phosphatidylserine externalization resulting in rescue and proliferation of cells.

On the other hand, supplementation with EPA inhibits apoptosis without additional protein content. Recently, Granci et al. demonstrated that administration of fish oil (mainly EPA) to mice with 5-FU may be a better alternative for increasing the efficiency of chemotherapic protocols through a BAX-dependent mitochondrial pathway [34]. As shown in our inflammatory cytokine data, EPA administration also resulted in decreased concentrations of IL- 6 and TNF- $\alpha$ compared to mice consuming the diet supplemented with DHA. These cytokines promote apoptosis; therefore, the effect of EPA on apoptosis signaling might account for the decrease in these cytokines. Furthermore, anti-apoptotic gene expression was significantly upregulated in all diets that had EPA suggesting again an a possible protective mechanism.

It is well known that the inhibition of tumor growth by EPA can be reversed by $n-6$ PUFA linoleic acid. This indicates an involvement of prostaglandin or lipoxygenase metabolites would induce anti-tumor action [35]. Wynter et al. reported that the anti-cachectic effect of EPA is only seen in joint with 5-FU, and that anticachectic treatment with EPA may be more beneficial when EPA is administrated during cycles of chemotherapy [36]. This might be expected, since EPA cannot be expected to counteract weight loss that is related to drug toxicity. According to our data and previous publications, EPA and fish oil act primarily on tumor suppression and inflammation related to the use of 5-FU, whereas DHA plays a key role in the suppression of 5FU induced intestinal impairment by repairing DNA. Furthermore, although our data suggests EPA and DHA have anti-apoptotic effects, which hypothetically may promote tumorgenesis, in vitro and in vivo studies have not shown any detrimental effects of EPA or DHA on chemotherapeutic agents [36-38].

Interestingly, Cont + FO diet also had a combination of EPA and DHA at higher concentrations then the EPA-DHA rich diet. Interestingly, Cont + FO did not result in as much protection as EPA-DHA rich diets alone. We hypothesize that this may likely be because FO contains only 10-20\% EPA and DHA and has a variety of other fatty acids including omega- 6 fatty acids which have been shown to promote tumor cell proliferation [39] and result in weight gain, tissue inflammation, and inhibition of EPA and DHA protective effects when taken in excess [40, 41].

Taken together our data suggests that a diet only rich in EPA and DHA demonstrated a protective synergistic effect against 5-FU induced intestinal impairment. These findings further demonstrate that a formula rich in EPA and DHA brings benefits to patients while undergoing chemotherapy and has future clinical implications. However, future clinical studies investigating human effects and changes on inflammation and intestinal impairment induced by cancer and chemotherapy are necessary as well as a more detailed analysis regarding the different roles of omega-3 fatty acids such as EPA and DHA.

\section{Conclusion}

The diet enhanced with EPA and DHA results in synergism protecting against the detrimental effects of 5-FU and limiting chemotherapy induced mucosal impairment. 


\section{Abbreviations}

5-FU: 5-fluorouracil; BCL-2: B-cell lymphoma 2; BID: BH3-interacting domain death agonist; DAO: diamine oxidase; DHA: docosahexaenoic acid; EPA: eicosapentaenoic acid; GALT: gut associated lymphoid tissue; IAP-1: inhibitor of apoptosis 1; IL-6: interleukin-6, SPF, specific pathogen-free; TMB: 3,3',5,5'tetramethylbenzidine; TNFa: tumor necrosis factor a.

\section{Competing interests}

The authors declare that they have no competing interests.

\section{Authors' contributions}

MS conducted research and wrote manuscript. RT designed research, conducted the research, wrote the manuscript, and has responsibility for the final content; SY and $\mathrm{NH}$ conducted research; HS, YTH and YN planed research and edited manuscript. TO, NK, KT edited manuscript. YMT provided essential reagents and analyzed data. All authors have read and approved the final manuscript.

\section{Acknowledgments}

We also thank Mariko Takegawa and Sayaka Sasaga for their technical support. Funding was provided by the Japan Society for the Promotion of Sciences, Tokyo JSPS KAKENHI 15 K16207 (R.T.) and 25462405 (Y.M.T)

\section{Author details}

Department of Nutrition and Metabolism, Institute of Biomedical Sciences, Tokushima University, 3-18-15 Kuramoto, Tokushima, Japan. ${ }^{2}$ Department of Anesthesiology, Institute of Biomedical Sciences, Tokushima University, Tokushima, Japan.

\section{Received: 10 October 2015 Accepted: 10 March 2016 Published online: 17 March 2016}

\section{References}

1. Stewart FM, Temeles D, Lowry P, Thraves T, Grosh WW, Quesenberry PJ. Post-5fluorouracil human marrow: stem cell characteristics and renewal properties after autologous marrow transplantation. Blood. 1993:81(9):2283-9.

2. Tisdale MJ. Cancer cachexia. Anticancer Drugs. 1993;4(2):115-25.

3. Spijkervet FK, Sonis ST. New frontiers in the management of chemotherapy-induced mucositis. Curr Opin Oncol. 1998;10 Suppl 1:S23-7.

4. Dikken C, Sitzia J. Patients' experiences of chemotherapy: side-effects associated with 5-fluorouracil + folinic acid in the treatment of colorectal cancer. J Clin Nurs. 1998;7(4):371-9

5. Tsuji E, Hiki N, Nomura S, Fukushima R, Kojima J, Ogawa T, et al. Simultaneous onset of acute inflammatory response, sepsis-like symptoms and intestinal mucosal injury after cancer chemotherapy. Int J Cancer. 2003;107(2):303-8

6. Gomez de Segura IA, Valderrabano S, Vazquez I, Vallejo-Cremades MT, Gomez-Garcia L, Sanchez M, et al. Protective effects of dietary enrichment with docosahexaenoic acid plus protein in 5-fluorouracil-induced intestinal injury in the rat. Eur J Gastroenterol Hepatol. 2004;16(5):479-85.

7. Nagayoshi H, Fukatsu K, Ueno C, Hara E, Maeshima Y, Omata J, et al. 5-Fluorouracil infusion reduces gut-associated lymphoid tissue cell number and mucosal immunoglobulin A levels. JPEN J Parenter Enteral Nutr. 2005:29(6):395-400.

8. Choi K, Lee SS, Oh SJ, Lim SY, Lim SY, Jeon WK, et al. The effect of oral glutamine on 5-fluorouracil/leucovorin-induced mucositis/stomatitis assessed by intestinal permeability test. Clin Nutr. 2007;26(1):57-62.

9. Lalla RV, Peterson DE. Treatment of mucositis, including new medications. Cancer J. 2006;12(5):348-54.

10. Fukatsu K, Nagayoshi H, Maeshima Y, Ueno C, Saitoh D, Mochizuki H. Fish oil infusion reverses 5 -fluorouracil-induced impairments in mucosal immunity in mice. Clin Nutr. 2008;27(2):269-75.

11. Kinoshita K, Ikai I, Gomi T, Kanai M, Tsuyuki S, Hirose T, et al. Exposure of hepatic sinusoidal mononuclear cells to UW solution in situ but not ex vivo induces apoptosis. J Hepatol. 1998:29(2):300-5.

12. Inomata A, Horii I, Suzuki K. 5-Fluorouracil-induced intestinal toxicity: what determines the severity of damage to murine intestinal crypt epithelia? Toxicol Lett. 2002;133(2-3):231-40.

13. Donaldson MS. Nutrition and cancer: a review of the evidence for an anti-cancer diet. Nutr J. 2004;3:19.
14. Nelson RL, Tanure JC, Andrianopoulos G, Souza G, Lands WE. A comparison of dietary fish oil and corn oil in experimental colorectal carcinogenesis. Nutr Cancer. 1988;11(4):215-20.

15. Lindner MA. A fish oil diet inhibits colon cancer in mice. Nutr Cancer. 1991;15(1):1-11.

16. Zock $\mathrm{PL}$, Katan MB. Linoleic acid intake and cancer risk: a review and meta-analysis. Am J Clin Nutr. 1998;68(1):142-53.

17. Pischon T, Hankinson SE, Hotamisligil GS, Rifai N, Willett WC, Rimm EB. Habitual dietary intake of $n-3$ and $n-6$ fatty acids in relation to inflammatory markers among US men and women. Circulation. 2003;108(2):155-60.

18. Johnson $\mathrm{GH}$, Fritsche K. Effect of dietary linoleic acid on markers of inflammation in healthy persons: a systematic review of randomized controlled trials. J Acad Nutr Diet. 2012:112(7):1029-41. 41 e1-15.

19. Reddy BS, Burill C, Rigotty J. Effect of diets high in omega-3 and omega-6 fatty acids on initiation and postinitiation stages of colon carcinogenesis. Cancer Res. 1991;51(2):487-91.

20. Culp BR, Titus BG, Lands WE. Inhibition of prostaglandin biosynthesis by eicosapentaenoic acid. Prostaglandins Med. 1979;3(5):269-78.

21. Takagi K, Nakao M, Ogura Y, Nabeshima T, Kunii A. Sensitive colorimetric assay of serum diamine oxidase. Clin Chim Acta. 1994;226(1):67-75.

22. Ruth MR, Field CJ. The immune modifying effects of amino acids on gut-associated lymphoid tissue. J Anim Sci Biotechnol. 2013;4(1):27.

23. Takechi H, Mawatari K, Harada N, Nakaya Y, Asakura M, Aihara M, et al. Glutamine protects the small intestinal mucosa in anticancer drug-induced rat enteritis model. J Med Invest. 2014;61(1-2):59-64.

24. Abimosleh SM, Tran CD, Howarth GS. Emu Oil: a novel therapeutic for disorders of the gastrointestinal tract? J Gastroenterol Hepatol. 2012;27(5):857-61.

25. Tazuke Y, Maeda K, Wasa M, Satoko N, Fukuzawa M. Protective mechanism of glutamine on the expression of proliferating cell nuclear antigen after cisplatin-induced intestinal mucosal injury. Pediatr Surg Int. 2011;27(2):151-8.

26. Ortega M, Gomez-de-Segura IA, Vazquez I, Lopez JM, de Guevara CL, De-Miguel E. Effects of growth hormone plus a hyperproteic diet on methotrexate-induced injury in rat intestines. Acta Oncol. 2001;40(5):615-21

27. Vazquez I, Gomez-de-Segura IA, Grande AG, Escribano A, Gonzalez-Gancedo P, Gomez A, et al. Protective effect of enriched diet plus growth hormone administration on radiation-induced intestinal injury and on its evolutionary pattern in the rat. Dig Dis Sci. 1999;44(11):2350-8.

28. Tsutsumi R, Horikawa YT, Kume K, Tanaka K, Kasai A, Kadota T, et al. Whey Peptide-Based Formulas With omega-3 Fatty Acids Are Protective in Lipopolysaccharide-Mediated Sepsis. JPEN J Parenter Enteral Nutr. 2015:39(5):552-61.

29. Vanderhoof JA, Blackwood DJ, Mohammadpour H, Park JH. Effects of oral supplementation of glutamine on small intestinal mucosal mass following resection. J Am Coll Nutr. 1992;11(2):223-7.

30. Wilmore DW. Glutamine and the gut. Gastroenterology. 1994;107(6):1885-6.

31. Souba WW, Klimberg VS, Copeland 3rd EM. Glutamine nutrition in the management of radiation enteritis. JPEN J Parenter Enteral Nutr. 1990;14(4 Suppl):106S-8

32. Calviello G, Palozza P, Maggiano N, Piccioni E, Franceschelli P, Frattucci A et al. Cell proliferation, differentiation, and apoptosis are modified by n-3 polyunsaturated fatty acids in normal colonic mucosa. Lipids. 1999;34(6):599-604

33. Geske FJ, Lieberman R, Strange R, Gerschenson LE. Early stages of p53-induced apoptosis are reversible. Cell Death Differ. 2001;8(2):182-91.

34. Granci V, Cai F, Lecumberri E, Clerc A, Dupertuis YM, Pichard C. Colon cancer cell chemosensitisation by fish oil emulsion involves apoptotic mitochondria pathway. Br J Nutr. 2013;109(7):1188-95.

35. Hudson EA, Tisdale MJ. Comparison of the effectiveness of eicosapentaenoic acid administered as either the free acid or ethyl ester as an anticachectic and antitumour agent. Prostaglandins Leukot Essent Fatty Acids. 1994;51(2):141-5.

36. Wynter MP, Russell ST, Tisdale MJ. Effect of $n-3$ fatty acids on the antitumour effects of cytotoxic drugs. In Vivo. 2004;18(5):543-7.

37. Schley PD, Jijon HB, Robinson LE, Field CJ. Mechanisms of omega-3 fatty acid-induced growth inhibition in MDA-MB-231 human breast cancer cells. Breast Cancer Res Treat. 2005:92(2):187-95.

38. Yang T, Fang S, Zhang HX, Xu LX, Zhang ZQ, Yuan KT, et al. N-3 PUFAs have antiproliferative and apoptotic effects on human colorectal cancer stem-like cells in vitro. J Nutr Biochem. 2013;24(5):744-53. 
39. Schley PD, Brindley DN, Field CJ. (n-3) PUFA alter raft lipid composition and decrease epidermal growth factor receptor levels in lipid rafts of human breast cancer cells. J Nutr. 2007;137(3):548-53.

40. Alvheim AR, Torstensen BE, Lin YH, Lillefosse HH, Lock EJ, Madsen L, et al. Dietary linoleic acid elevates the endocannabinoids 2-AG and anandamide and promotes weight gain in mice fed a low fat diet. Lipids. 2014;49(1):59-69.

41. Alvheim AR, Torstensen BE, Lin YH, Lillefosse HH, Lock EJ, Madsen L, et al. Dietary linoleic acid elevates endogenous 2-arachidonoylglycerol and anandamide in Atlantic salmon (Salmo salar L.) and mice, and induces weight gain and inflammation in mice. Br J Nutr. 2013;109(8):1508-17.

Submit your next manuscript to BioMed Central and we will help you at every step:

- We accept pre-submission inquiries

- Our selector tool helps you to find the most relevant journal

- We provide round the clock customer support

- Convenient online submission

- Thorough peer review

- Inclusion in PubMed and all major indexing services

- Maximum visibility for your research

Submit your manuscript at www.biomedcentral.com/submit
Biomed Central 